\title{
Parabolic Focal Conics and Polygonal Textures in Lipid Liquid Crystals
}

\section{Citation}

Asher, S. A. and Peter S. Pershan. 1979. Parabolic focal conics and polygonal textures in lipid liquid crystals. Journal de Physique 40(2): 161-173.

\section{Published Version}

doi:10.1051/jphys:01979004002016100

\section{Permanent link}

http://nrs.harvard.edu/urn-3:HUL.InstRepos:10361925

\section{Terms of Use}

This article was downloaded from Harvard University's DASH repository, and is made available under the terms and conditions applicable to Other Posted Material, as set forth at http:// nrs.harvard.edu/urn-3:HUL.InstRepos:dash.current.terms-of-use\#LAA

\section{Share Your Story}

The Harvard community has made this article openly available.

Please share how this access benefits you. Submit a story.

\section{Accessibility}




\title{
Parabolic focal conics and polygonal textures in lipid liquid crystals $(*)$
}

\author{
S. A. Asher and P. S. Pershan \\ Division of Applied Sciences, Gordon McKay Laboratory, Harvard University, Cambridge, Massachusetts 02138, U.S.A.
}

(Reçu le 10 juillet 1978, accepté le 24 octobre 1978)

\begin{abstract}
Résumé. - Les phases liquide-cristallines lyotropes de dipalmitoyl-, dimyristoyl- et dilaurylphosphatidylcholine préparées pour différentes concentrations en eau présentent des réseaux de défauts polygonaux que l'on étudie au microscope optique. Les réseaux sont semblables à ceux observés précédemment dans le cas de phases thermotropes smectiques et cholestériques. Le modèle de domaine focal parabolique proposé par Rosenblatt, Pindak, Clark et Meyer pour les réseaux des smectiques thermotropes permet aussi de rendre compte des propriétés observées dans ces phases lamellaires lyotropes. La biréfringence des réseaux polygonaux s'accroît progressivement avec les contraintes qui induisent les défauts. Le modèle de domaine focal parabolique ne décrit pas complètement les détails des réseaux polygonaux les plus biréfringents.
\end{abstract}

\begin{abstract}
Polygonal defect arrays in lipid liquid crystals prepared from dipalmitoyl-, dimyristoyl-, and dilaurylphosphatidylcholine with various concentrations of water have been observed and characterized using optical microscopy. These arrays appear similar to the polygonal arrays previously observed in thermotropic smectic and cholesteric liquid crystals. The parabolic focal conic model proposed by Rosenblatt, Pindak, Clark and Meyer for the polygonal arrays in thermotropic smectic liquid crystals also describes the observed properties in the smectic A phase of lipid liquid crystals. The polygonal arrays become progressively more birefringent as the strains inducing them are increased. The parabolic focal conic model does not completely describe the details of the more birefringent polygonal arrays.
\end{abstract}

1. Introduction. - Aligned monodomain lipid multilayers are of interest not only because of their liquid crystalline properties, but also because of their utility as model systems in the study of biological membranes $[1,2]$. These lipid liquid crystals can be utilized either directly for the study of the material properties of lipid bilayers [3-13] or, with the incorporation of biologically interesting ingredients [12], to study the properties of these ingredients in the model system. If one can incorporate proteins in aligned lipid multilamellae, these systems would be uniquely suited for study of the conformational properties of the proteinlipid system.

For any of these studies it is advantageous to have large, monodomain samples of the size that were recently obtained by Powers et al. [12, 13]. They described a technique by which lipid-water mixtures

(*) This work has been supported in part by the Joint Services Electronics Program (U.S. Army, Navy, and Air Force) under Contract Number N00014-75-C-0648 and by the National Science Foundation under Grant Number DMR-76-01111 and DMR76-22452. containing less than $8 \%$ by weight of water could be annealed into aligned monodomain liquid crystals $\left(\sim 100 \mu \mathrm{m}\right.$ thick and $\left.1 \mathrm{~cm}^{2}\right)$ at elevated temperatures $\left(>100^{\circ} \mathrm{C}\right)$. We have used this technique to prepare aligned samples of dipalmitoyl- (DPPC), dimyristoyl(DMPC) and dilauryl- (DLPC) phosphatidylcholine containing up to $8 \%$ water. We have also developed a mechanical alignment technique for the room temperature alignment of samples of DLPC containing $20 \%$ water. With both of these techniques and with all three of these lipids we have observed various defect structures [14]. One of these structures is qualitatively similar to polygonal arrays, previously observed in cholesteric [15] and thermotropic smectic liquid crystals [16-18]. We have characterized these arrays by optical microscopy and propose a structure for one of them that is identical to the parabolic focal conic (PFC) model proposed initially by Rosenblatt et al. [18] to explain the polygonal arrays that they observed in a thermotropic smectic liquid crystal. We also demonstrate the existence of arrays that have many properties in common with the PFC, but which are distinctly different. 
2. Experimental. - Dipalmitoyl and dimyristoyl phosphatidylcholine (DPPC and DMPC) were purchased from Sigma Chemical Co. (St. Louis, MO, USA). Dilauryl phosphatidylcholine (DLPC) was purchased from Calbiochem (San Diego, CA, USA). Prior to use, each of the samples was monitored for purity by thin layer chromatography. We have found experimentally that lipids lyophilized from benzene align more easily and with fewer defects than if used directly. This may be due to the fact that lipids dissolved in benzene form large inverse micelles that have a high degree of local order [19]. DPPC, DMPC and DLPC were aligned homeotropically (smectic layers are parallel to the glass surfaces) between $1 \mathrm{~mm}$ thick microscope slides separated by $125 \mu \mathrm{m}$ spacers using the Powers technique $[12,13]$. We found, however, that if the glass slides were scrupulously clean, alignment was obtained equally well with or without the silane surfactant described by Powers.

The hydrophilic glass surfaces were prepared by cleaning the slides with hot chromic acid and rinsing them in a distilled water steam bath; the condensing water rinsed the glass surfaces. In view of the amphiphilic character of lipids, it is not surprising that a hydrophilic glass surface would align samples just as well as the hydrophobic surface obtained with the surfactant.

For water concentration of less than $10 \%$ by weight, the water was added directly to the lipid. Samples of DLPC with $20 \%$ water were prepared by adding the water to the lipid and dispersing it using a dental amalgamator. Immediately after the addition of water to the lipid, the sample was tightly clamped to prevent the escape of water during the annealing process. We estimate the reported water concentration to be accurate to $\pm 10 \%$ of the value quoted. The aligned samples of DPPC appeared homogeneously black, with few defects when observed microscopically between crossed polarizers. The aligned samples of DLPC with $20 \%$ water had thin strand-like defects that separated homogeneous black domains (typically $3 \mathrm{~mm}^{2}$ in area). In any one experiment identical effects were observed in the separate domains. Although the temperatures at which phase transitions occur in lipid-water systems are strong functions of the water concentration $[1,12,20,21]$, none of the detected features of the defect structure were dependent on the water concentration so long as the sample was maintained in the $L \alpha$ or smectic A phase.

Samples of DLPC with $20 \%$ water were aligned at room temperature by placing the lipid-water mixture between glass slides separated by a $100 \mu \mathrm{m}$ latex rubber spacer. The holder for the slides (Fig. 1) consisted of a metal box and a steel plate which compressed the glass slides together and against the bottom of the box. The tension on the steel plate was varied by set screws accessible from the top of the sample holder. Holes were drilled in the sample holder to monitor the alignment. Macroscopic align-

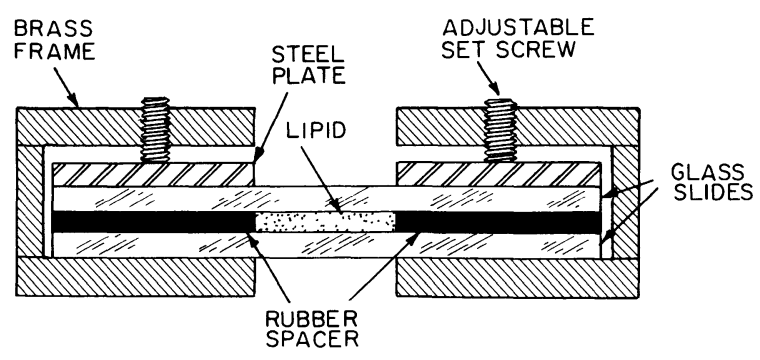

Fig. 1. - Sample holder for the mechanical alignment of lipids.

ment of DLPC containing $20 \%$ water could be obtained at room temperature by gently alternating between compressing and releasing the glass slides. In general the compression was non-uniform, and flow from one part of the sample to another could be observed during the process. We presume that this flow is responsible for the growth of the aligned homeotropic areas as the process is continued. The alignment and defect structures were monitored continuously using polarized optical microscopy and conoscopy. The phase shift in the transmitted light induced by the sample was measured at selected points in the sample by a Soleil-Babinet compensator attached to the microscope.

3. Results. Well aligned, homeotropic (layers are parallel to glass surfaces) samples of DPPC, DMPC, and DLPC with various amounts of water (4 to $20 \%$ ) at temperatures which correspond to their smectic $A$ or $L \alpha$ phase are uniaxial $[12,13]$. The structure of these lipid liquid crystals has been shown by X-ray diffraction to consist of well-defined bilayers of lipid molecules separated by layers of water (Fig. 2)

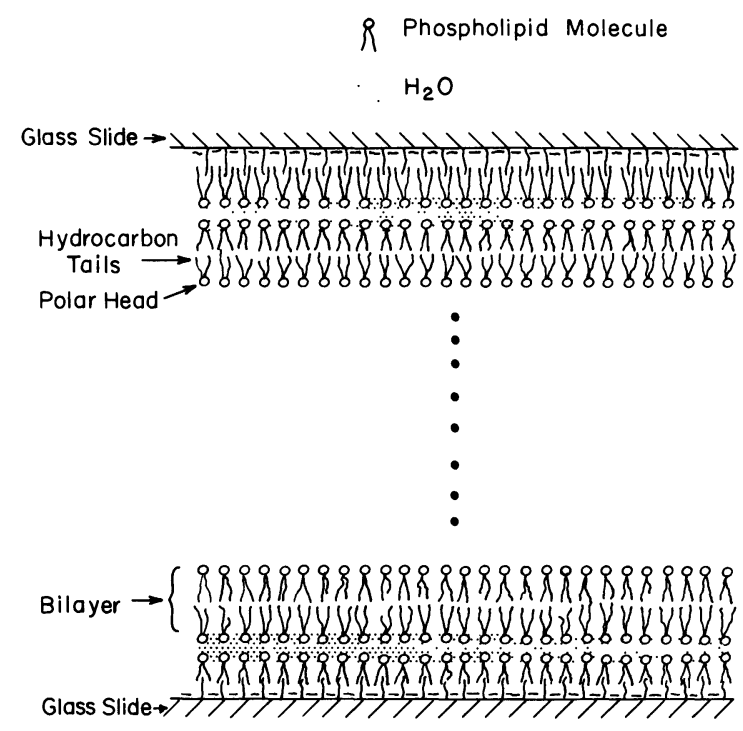

Fig. 2. - Schematic representation of the molecular geometry of lipid liquid crystals.

$[8,11,13,21-23]$. Although there is a well-defined order perpendicular to the layer planes, there appears to be no long-range order within the lipid layers 
$[20,22,23]$. Since the optic axis of the homeotropically aligned lipid in the $\mathrm{L} \alpha$ phase is normal to the glass surfaces, a well aligned sample appears uniformly dark when viewed between crossed polarizers. Defects in

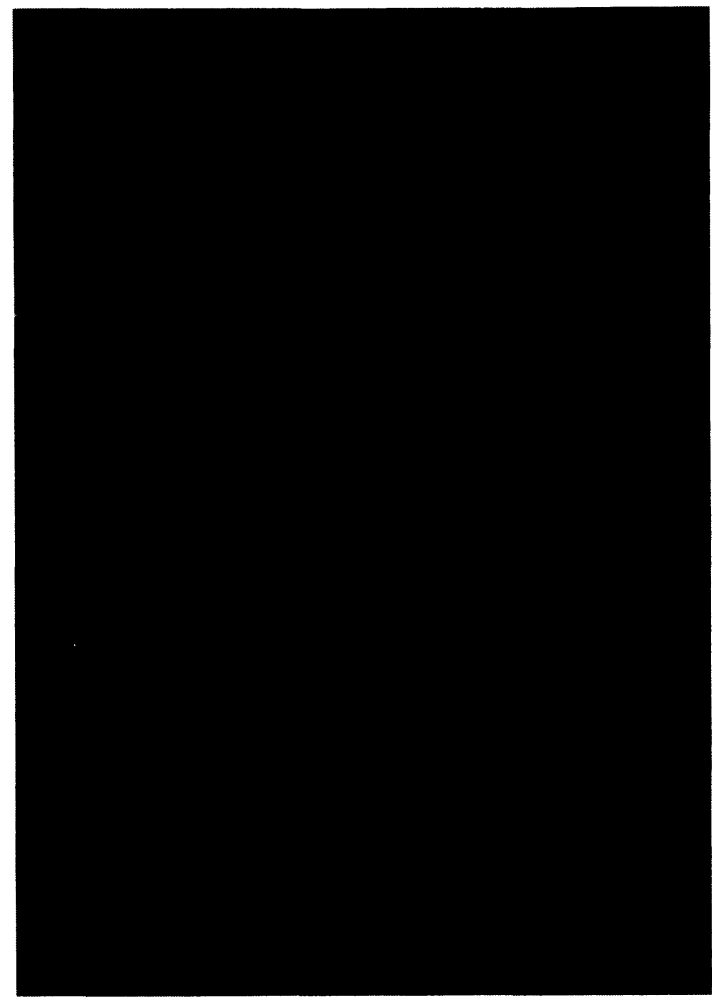

$\rightarrow 50 \mu \mathrm{m}$

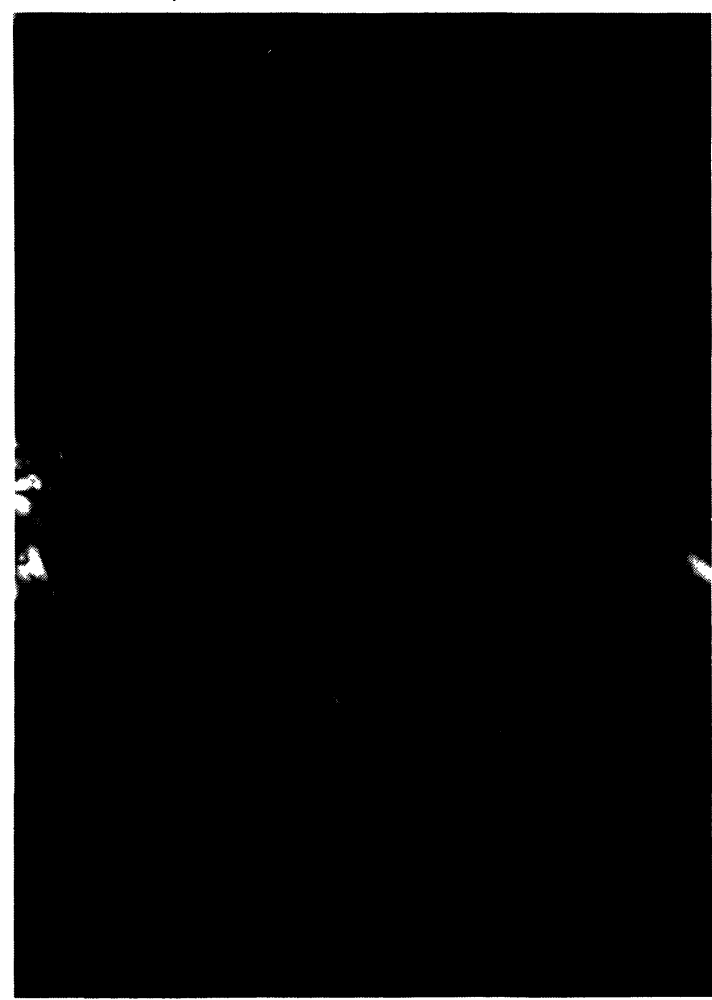

$b$

Fig. 3. - Photomicrograph between crossed polarizers of an aligned DPPC liquid crystal containing $7 \%$ water by weight. Sample is $125 \mu \mathrm{m}$ thick. a) Uniaxial sample. No light is transmitted. sample alignment, accompanied by tilts of the layers with respect to the glass slides, make the samples appear birefringent, such that when the plane of polarization of the incident light is neither parallel nor
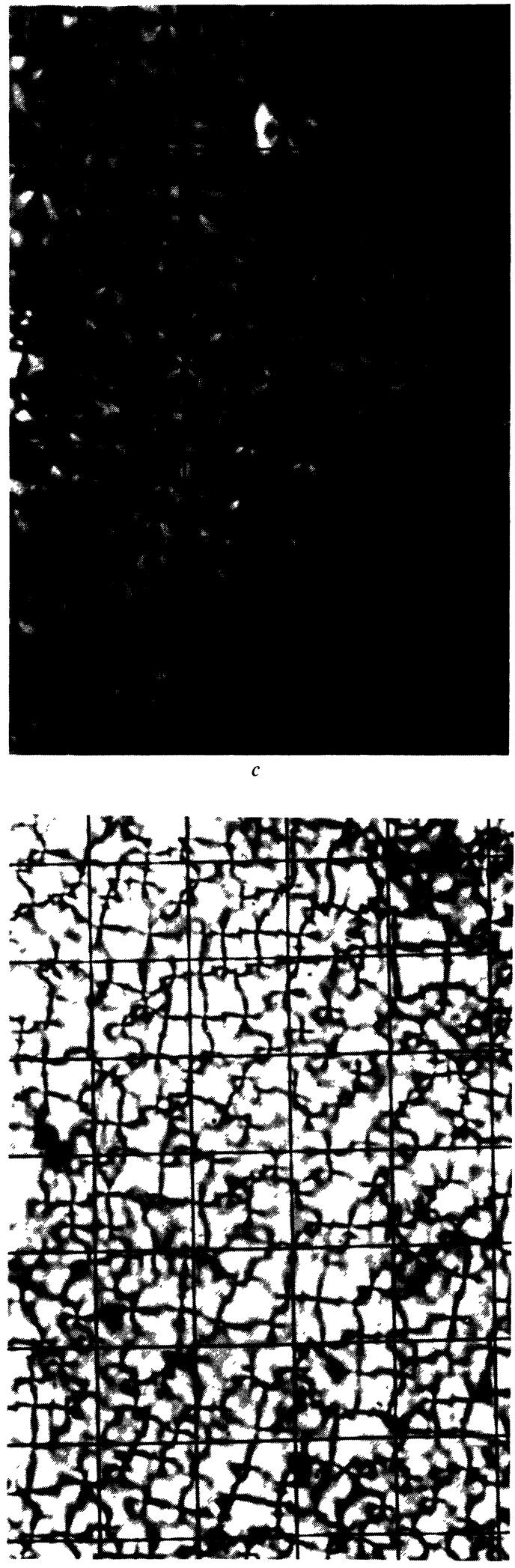

$T=72.5^{\circ}$.b) $T=73^{\circ} \mathrm{C}$. An array begins to appear. c) $T=73.5^{\circ} \mathrm{C}$. Array is clearly visible. $d) T=74^{\circ} \mathrm{C}$. Moderately birefringent array visible. 
perpendicular to the tilt direction the light leaving the sample will be elliptically polarized and will not be extinguished by a crossed polarizer. The magnitude of the effect is related to the amount of tilt, the magnitude of the uniaxial optical anisotropy of the aligned system, and the length of the tilted region as measured parallel to the direction of light propagation, i.e., normal to the glass slides. Figure $3 a$ shows an aligned monodomain sample of DPPC with $7 \%$ water by weight at $76^{\circ} \mathrm{C}$. Upon increasing the temperature slightly ( $<1^{\circ} \mathrm{C}$ within a few seconds) an array of polygonal defects is produced whose birefringence (and visibility) is a function of the temperature increase (Figs. $3 b-3 d$ ). This array is stable for hours and appears for each of the three lipids studied. The formation of the array is completely reversible ; upon lowering the temperature it disappears. Similarly, these polygonal arrays can also be formed by dilating the sample (pulling apart the glass plates enclosing the sample). When the stress is relieved by restoring the plates to their initial position, the array disappears. The fact that similar effects are produced for both a mechanical dilation and a temperature increase can be rationalized from the negative thermal expansion coefficient of lipid liquid crystals perpendicular to their layer planes [24, 25].

Some of the particular properties of the polygonal arrays, when viewed between crossed polarizers, are shown in figures $4 a, b$, and $c$. The three pictures were obtained by focusing the microscope to the three different levels within the sample where details of the arrays were in best focus. The crossed polarizers are parallel (perpendicular) to the reticule lines. There appear to be three related arrays, composed of black intersecting lines. The middle array (Fig. 4b) has a period of about $10 \mu \mathrm{m}$ for DPPC at $7 \%$ water and always appears exactly half way between the glass sides. The top and bottom arrays are symmetrically displaced above and below the middle array and have a period twice that of the middle array, about $20 \mu \mathrm{m}$. The displacement in height for the best focus of the top and bottom arrays from the position of best focus for the middle array is a function of the birefringence of the sample; the more birefringent the sample, the greater their displacements. For the sample shown in figure 4 the top (bottom) array is in sharpest focus $20 \mu \mathrm{m}$ above (below) the centre of the sample. Upon lowering the focus from the top of the sample, the points of intersection of the top array comes into focus first. As the focus is lowered further, the intersections of the lines of the top array go out of focus and the focus travels toward the sharply focused intersections of the middle array. As the focus is lowered still further toward the bottom array, the sharpness of the black lines travels toward the intersections of the bottom array. It appears as if the black lines occur in two perpendicular sets with the upper one concave upward and the lower one concave downward.
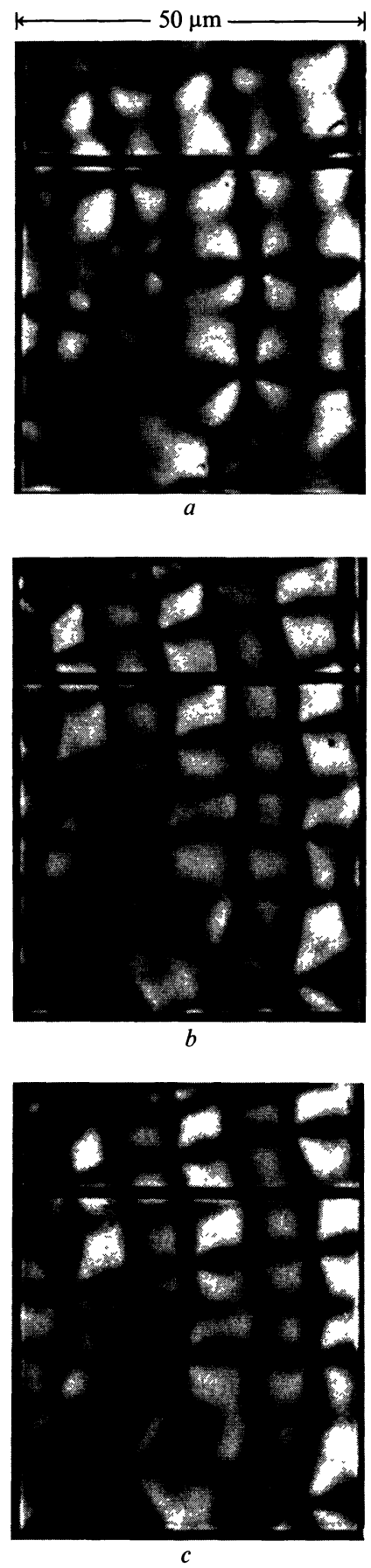

Fig. 4. - Photomicrograph between crossed polarizers of DPPC containing $7 \%$ water. $125 \mu \mathrm{m}$ thick. $T=76.5{ }^{\circ} \mathrm{C}$. a) Top array. Focus is $20 \mu \mathrm{m}$ above the mid-height of the sample. $b$ ) Middle array. Focus is at the mid-height of the sample. c) Bottom array. Focus is $20 \mu \mathrm{m}$ below the mid-height of the sample.

When the crossed polarizers are rotated with respect to the sample, the crosses at the intersections in the top and bottom arrays rotate along with the polarizers. This is shown in figures $5 a$ and $5 b$ for a small counterclockwise rotation. However, the crosses formed by the sharply focused intersections of the middle array rotate in the opposite direction from the direction of rotation of the crossed polarizers (Figs. $5 c$ and $5 d$ ). All of the polygonal arrays observed exhibit the above 

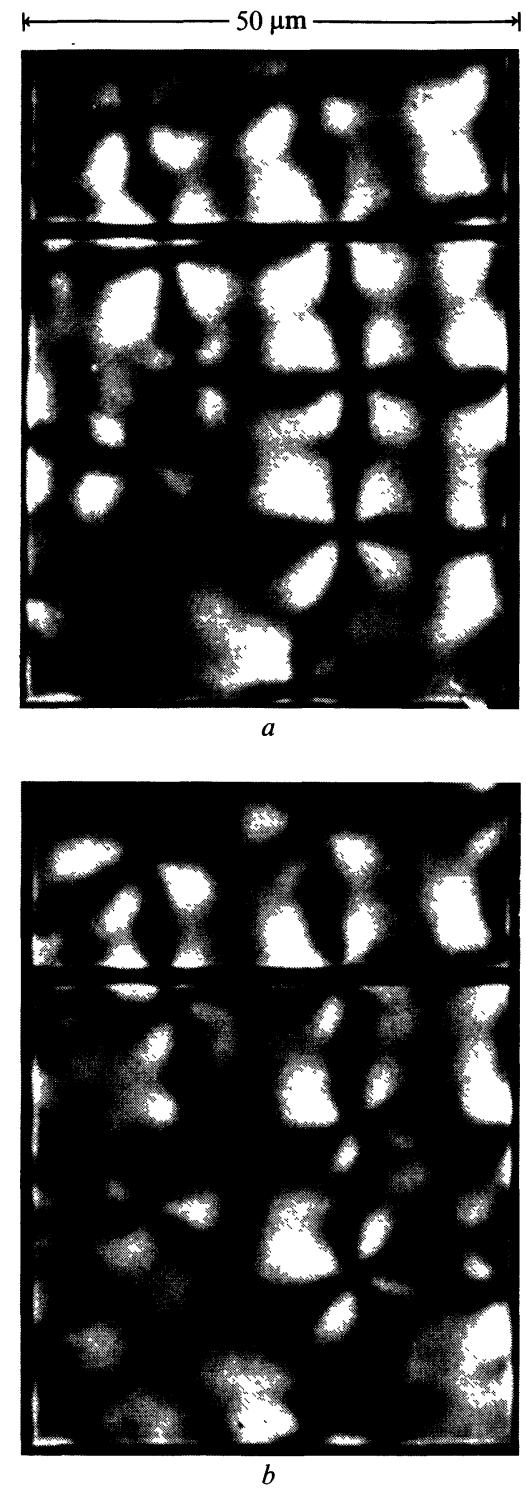

Fig. 5. - Effect of rotation of crossed polarizers on the intersections in the polygonal arrays of figure 4. a) Top array between crossed polarizers. $b$ ) Same as (a), but for a small counterclock

properties when observed with crossed polarizers. However, the more birefringent arrays have extra fine structure superposed on them, as shown in figure 7 below.

Additional information on the arrays was derived from dark field microscopy in which the sample is illuminated such that the only light that enters the objective is the light that has been scattered by optical inhomogeneities in the sample. Figure $6 a$ shows the polygonal array in a liquid crystal of DLPC with $20 \%$ water as viewed between crossed polarizers when the microscope is focused halfway between the glass slides. In different regions of the sample the array exhibits different degrees of birefringence. Figure $6 b$ shows the same sample viewed under dark field microscopy. A comparison of the dark field and crossed polarizer microscopy on the weakly birefringent arrays indicates that crosses occur in the dark
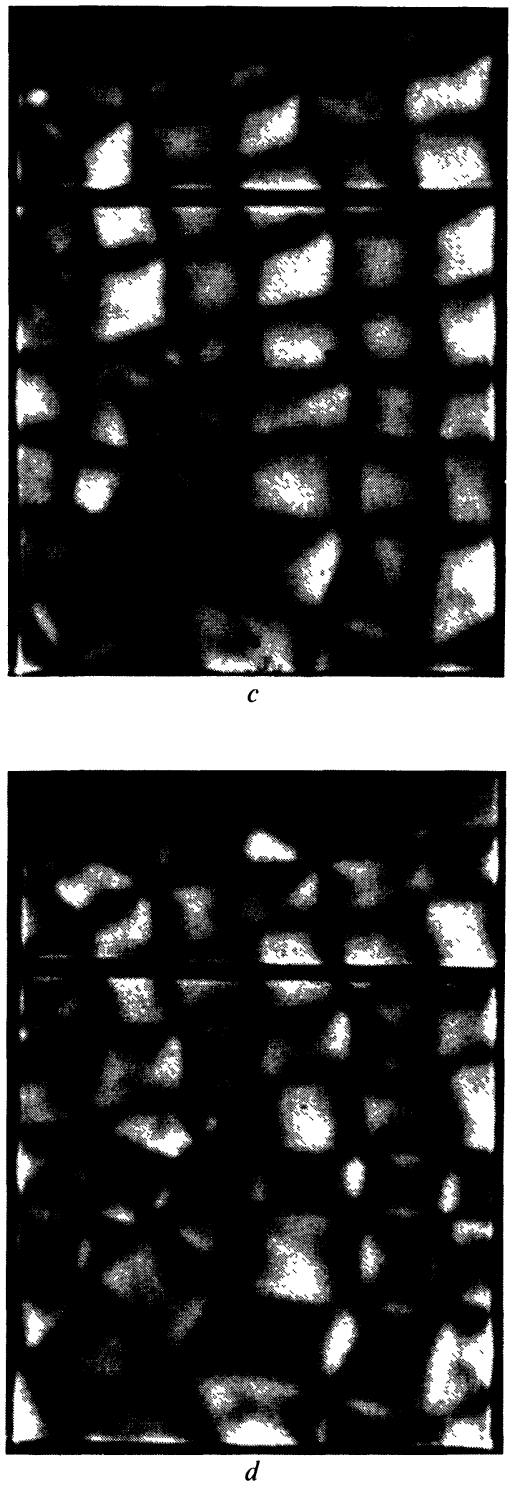

rotation of the crossed polarizers by $25^{\circ} . c$ ) Middle array between crossed polarizers. $d$ ) Same as $(c)$, but crossed polarizers rotated as in $(b)$.

field precisely at the sharply focused intersections of the middle array. For the weakly birefringent arrays, these were the only features observed under dark field conditions and were only observed to be in focus exactly halfway between the glass slides. The two perpendicular arms of the crosses observed in the dark field point toward the nearest intersections in the top and bottom arrays. One arm of the crosses extends along a straight line connecting two intersections of the top array, while the orthogonal arm extends along a line connecting two intersections of the bottom array.

Additional fine structure is observed when a strongly birefringent array is viewed between crossed polarizers (Fig. 7). As in the less birefringent arrays, three levels are observed and the crosses at the intersection of the top and bottom arrays rotate in the same direction as the crossed polarizers. The sharply focused crosses 

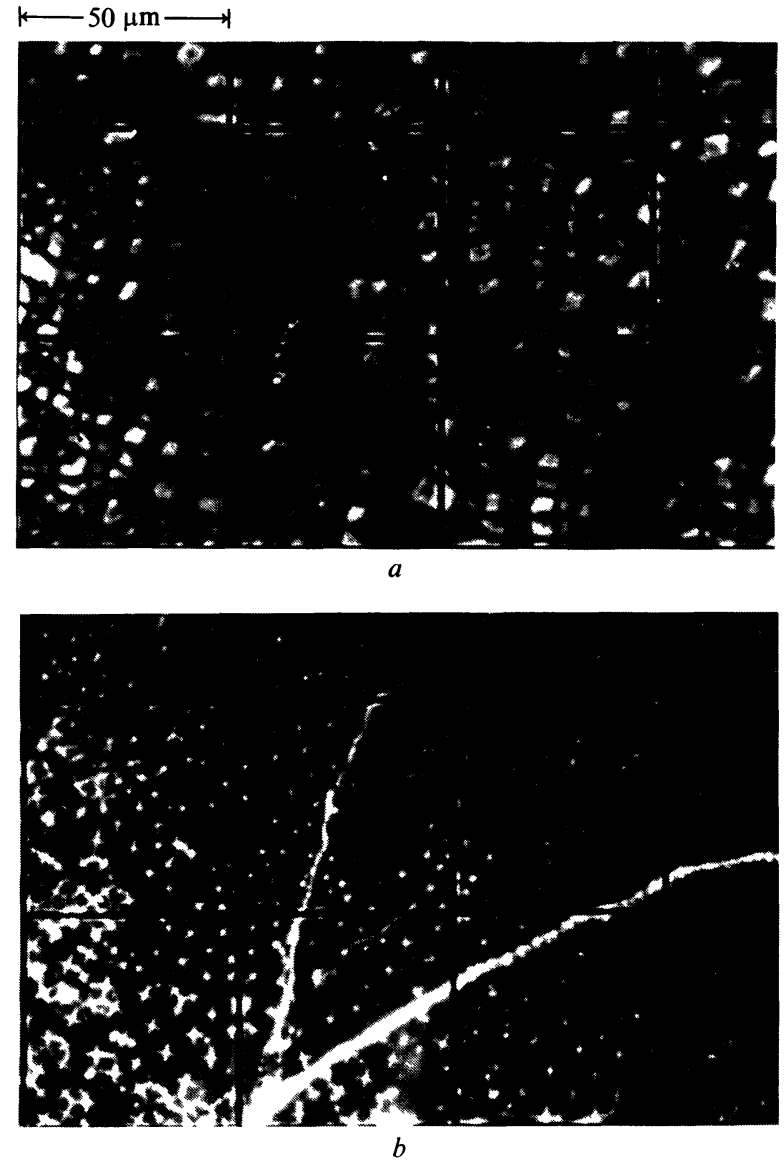

Fig. 6. - Crossed polarizers and dark field microscopy of polygonal arrays in liquid crystals of DLPC with $20 \%$ water. Room temperature. Sample thickness is $80 \mu \mathrm{m} . a$ ) Crossed polarizers. $b$ ) Dark field.

in the middle of the array rotate in the opposite direction. Dark field microscopy indicates a very complex structure for the array (Fig. 8). As the focus is lowered from the top of the sample, the first feature to come into focus is a hazy circle which has some substructure (Fig. 8a). The circle is located where the intersections of the top array are observed between crossed polarizers. As the focus is lowered, concentric circles of dots appear (Fig. 8b). At the mid-height, crosses are observed centred at the location of the intersections of the middle array (compare figures $7 a$ and $8 c$ ). As the focus is lowered further, a series of concentric circles again comes into focus, ending in a hazy circle located at the intersections of the bottom array, as viewed under crossed polarizers (Figs. $8 d$ and $8 e$ ).

The size and the birefringence of the polygonal arrays increase as the stresses inducing them increase. Small temperature rises $\left(\lesssim 0.5^{\circ} \mathrm{C}\right)$ result in a small expansion of the polygonal arrays and in an increase in their birefringence. However, for larger temperature rises $\left(0.5-1.0^{\circ} \mathrm{C}\right)$ the expansions are followed by an abrupt doubling of the period of the array. The change in the period of the array occurs very quickly $(<1 \mathrm{~s})$. Figure 9 shows two sets of polygonal arrays in the same sample. The less birefringent array has a period half that of the more birefringent array.
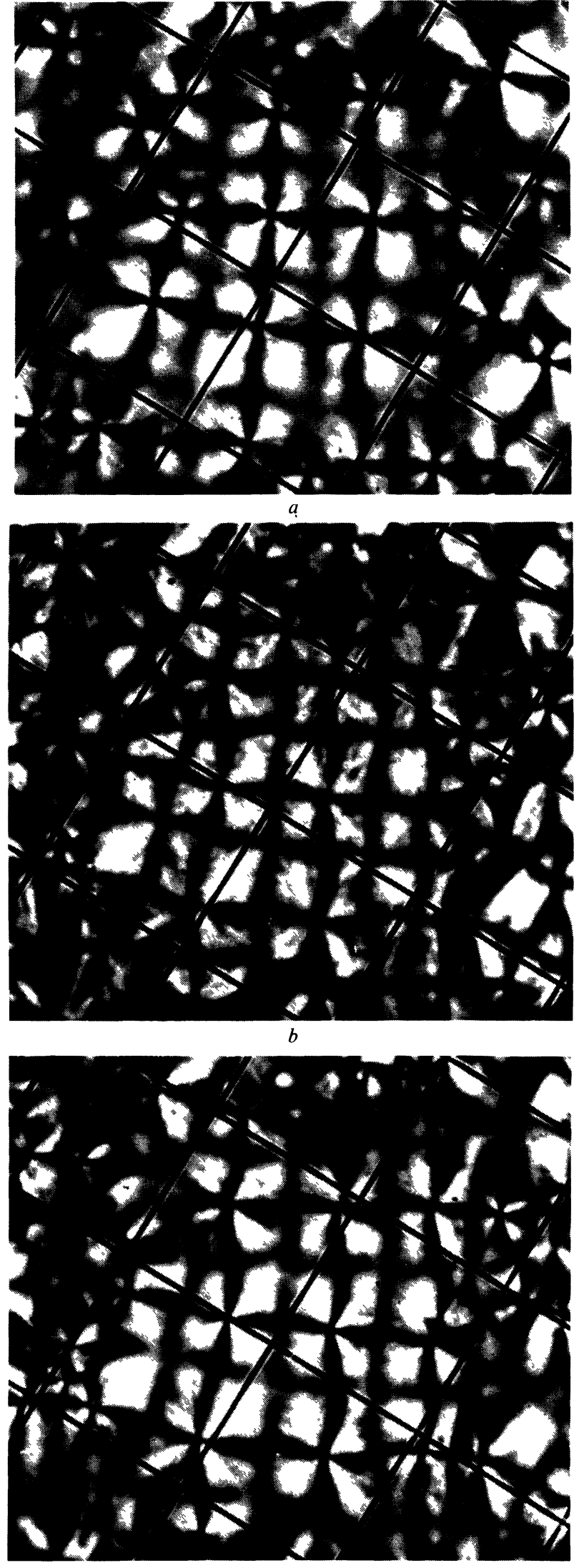

Fig. 7. - Photomicrograph between crossed polarizers of very birefringent polygonal arrays in DPPC with $20 \%$ water. Room temperature. Sample thickness is $85 \mu \mathrm{m}$. Reticule lines are $50 \mu \mathrm{m}$ apart. a) Top array. Focused $30 \mu \mathrm{m}$ above centre of sample. b) Middle array. Focused at mid-height of sample. $c$ ) Bottom array. Focused $30 \mu \mathrm{m}$ below middle of sample. 

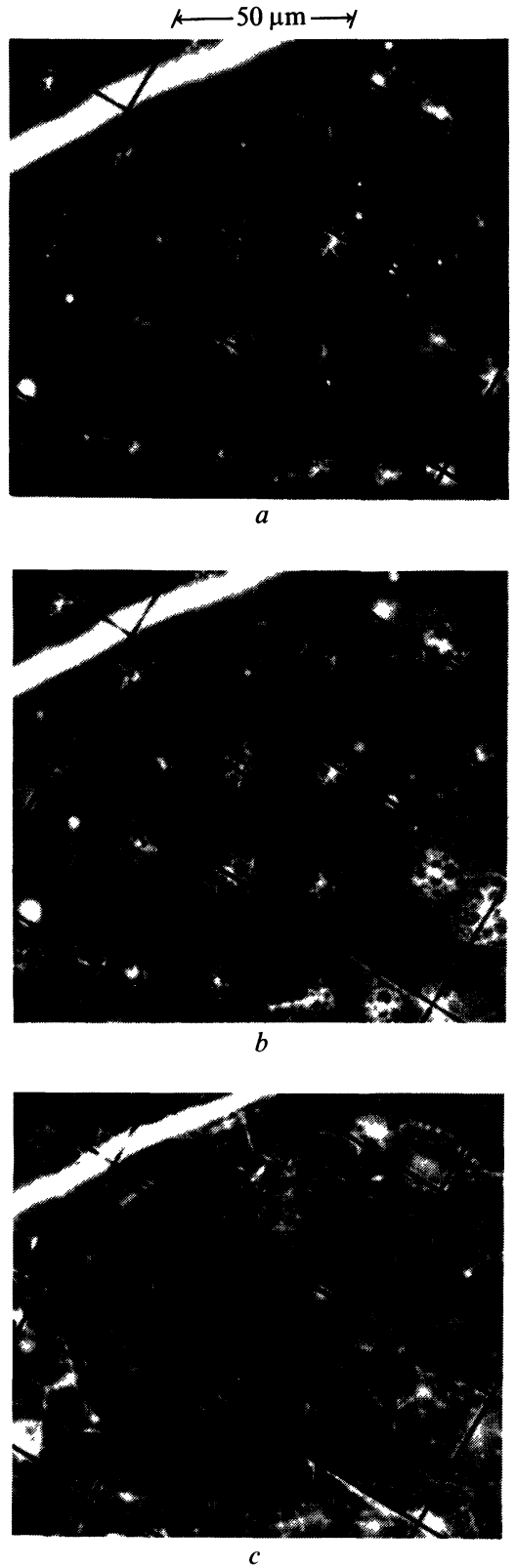

Fig. 8. - Photomicrographs using dark field of the sample shown in figure 7. Reticule lines are $50 \mu \mathrm{m}$ apart. a) Focused $30 \mu \mathrm{m}$ above centre of sample. $b$ ) Focused $20 \mu \mathrm{m}$ above centre of sample. $c$ ) Focused at centre of sample. $d$ ) Focused at $20 \mu \mathrm{m}$ below centre of sample. e) Focused at $30 \mu \mathrm{m}$ below centre of sample.

The weakly birefringent arrays cannot be seen microscopically under natural illumination. However, a faint pattern of dots appears for the more birefringent arrays. Figures $10 a, 10 b$, and $10 c$ show the dot pattern for the arrays shown in figures 7 and 8 . The intersections of the top and bottom arrays are poorly resolved as fuzzy black dots. The intersections of the middle array appear as black dots when focused above the middle array. These dots become white when focused at or below the middle of the sample. The dot pattern is difficult to observe in all but the more birefringent arrays. Subtle variations in detail occur depending on the birefringence of the array. Often
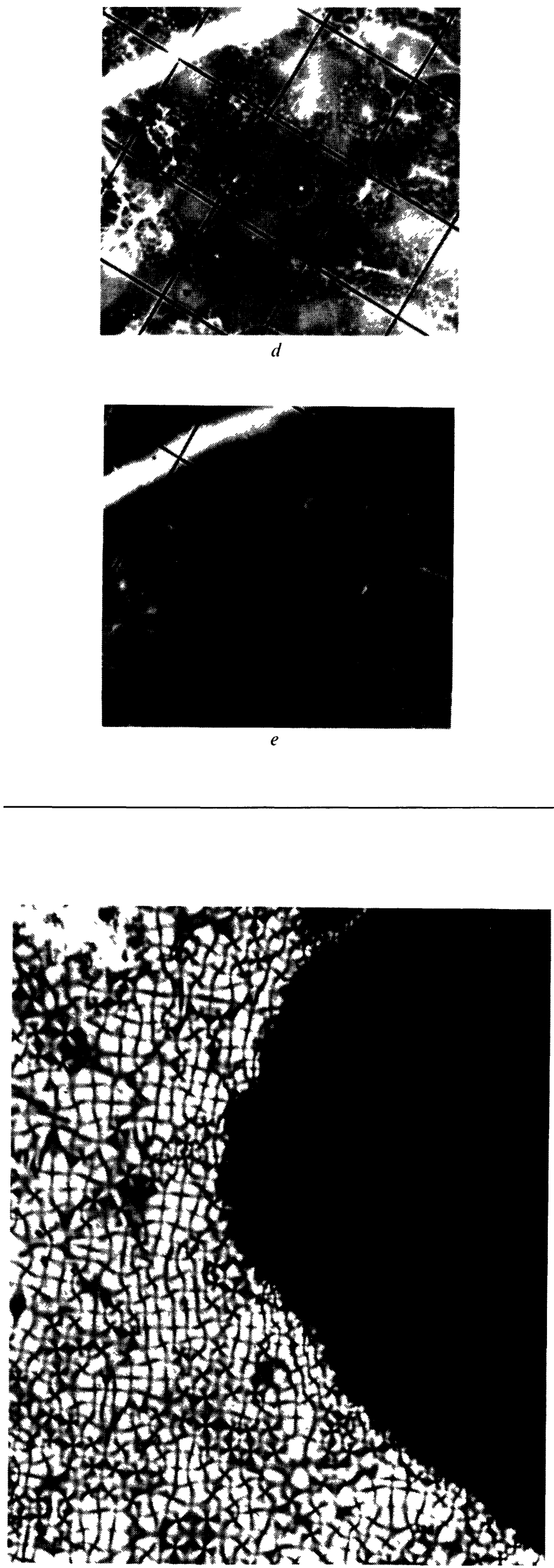

Fig. 9. - Photomicrograph between crossed polars of an aligned DPPC liquid crystal containing $7 \%$ water by weight at $96^{\circ} \mathrm{C}$. The sample thickness is $125 \mu \mathrm{m}$. The lighter, more birefringent arrays have a period twice that of the dark arrays. Focus is mid-height. 


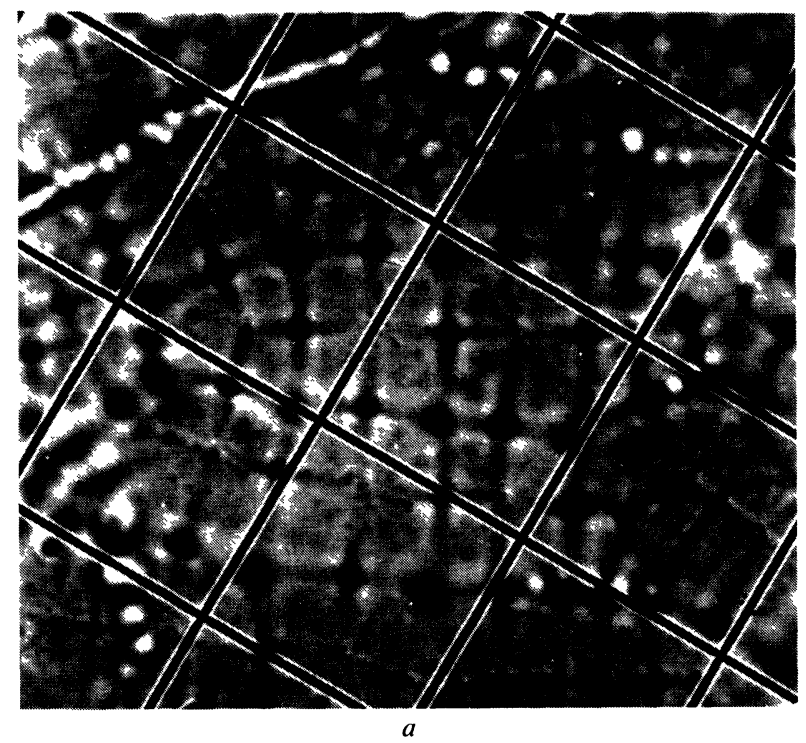

a faint series of lines between the dots is observed (Fig. 10).

If the crossed polarizers used to view the polygonal array shown in figure 4 are rotated by $45^{\circ}$ with respect to the sample, the dark lines in figure 4 are replaced by a network of white lobes connecting the intersections of the top, middle and bottom arrays (Fig. 11). Small black crosses are observed oriented along the polarizer axes at each of the intersections. Figure $11 b$
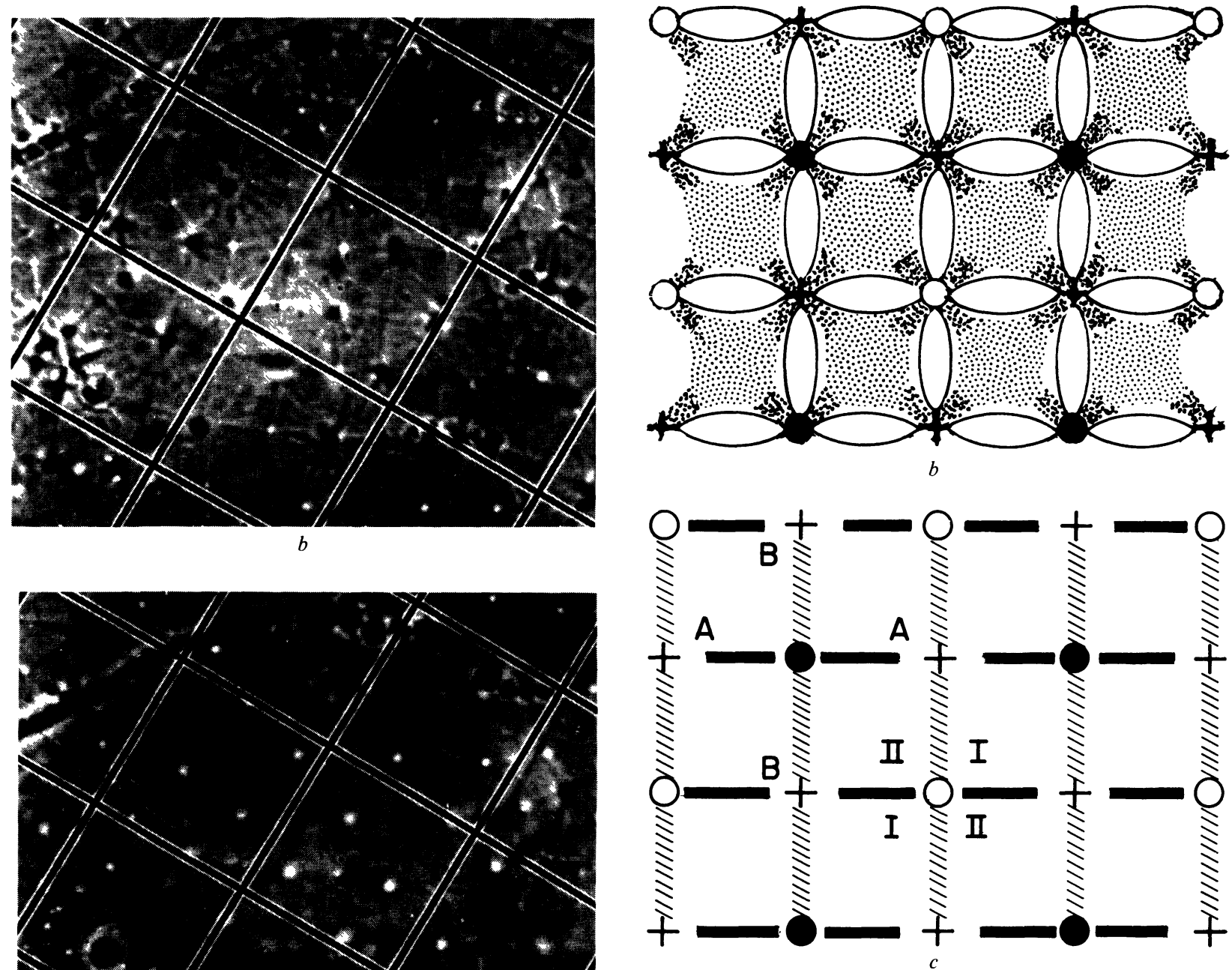

Fig. 11. - a) Sample of DPPC with $7 \%$ water seen in figures 4 and 5. The sample is viewed with crossed polarizers rotated by $45^{\circ}$ from that in figure 4. b) Schematic representation of sample shown in (a). Closed circles represent intersections of top array. Open circles represent intersections of bottom array. Crosses represent the intersections in the middle array whose crosses rotate in the opposite direction from the crossed polarizers (see Figs $5 c$ and $5 d$ ). c) Pattern observed with Soleil-Babinet compensator when the phase shift along the horizontal set of white lobes in $(a)$ and $(b)$ is compensated.

Fig. 10. - Photomicrographs using natural illumination of the sample shown in figures 7 and 8 . Reticule lines are $50 \mu \mathrm{m}$ apart. a) Focused $30 \mu \mathrm{m}$ above centre of sample. $b$ ) Focused in middle of sample. c) Focused $30 \mu \mathrm{m}$ below centre of sample. 
schematically represents the pattern observed in figure $11 a$. The closed black circles are the intersections of the top array, the open circles are the intersections of the bottom array, and the crosses are the sharply focused intersections of the middle array. Figure $11 c$ illustrates the results observed when a Soleil-Babinet compensator, oriented with its optic axis parallel to the white lobes, is inserted between one of the polarizers and the sample. The phase shift of the compensator can be adjusted to exactly compensate the sample birefringence along extended segments of the horizontal lines running through the intersections in either the top or bottom array and the sharply focused intersections of the middle array. The intersections themselves are uniaxial and will thus not be dark for this same setting of the compensator. Aside from this point, the line segments AA are observed to be uniformly dark. The largest birefringence in the sample occurs along these lines and the slow axis is parallel to AA.

For this same setting of the compensator, the vertical cross hatched lines, BB, running through the same intersections become brighter. The first and third quadrants, I, surrounding these intersections appear identically, dark, but not as dark as the lines AA, while the second and fourth quadrants, II, are noticeably lighter, but not as light as the lines BB. The array is also symmetrical, in the sense that rotation of the compensator by $90^{\circ}$ will cause the fine dark lines to appear along BB and the bright lines along AA.

Measurements of the ellipticity of the transmitted light along the dark segments AA obtain optical path differences of $15,60,80$ and $340 \mathrm{~nm}$ for the polygonal arrays shown in figures $3 b, 3 c, 4$ and 7 , respectively. The optical path differences appear constant along these lines. However, at the points where the lines AA and $\mathrm{BB}$ would intersect, the optical path differences are smaller. The optical path difference is also smaller away from these lines.

In contrast to the polygonal arrays shown in figures 3 and 4, which show little variation in the optical path difference at points away from the lines connecting the intersections of the top, middle, and bottom arrays, the polygonal array in figure 7 shows variations in the optical path difference at points away from these lines. These variations are difficult to measure due to the finite resolving power of the microscope. They are probably related to the corrugated ribbed pattern emanating from the intersections of the top and bottom arrays seen between crossed polarizers in figure 7 .

4. Discussion. - Recently, Rosenblatt et al. [18] described a model for defect structures in the thermotropic smectic A liquid crystal formed from cyanobenzylidene octyloxyaniline (CBOOA). CBOOA readily aligns both homogeneously (smectic layers perpendicular to glass surfaces) and homeotropically. They showed that CBOOA homogeneously aligned

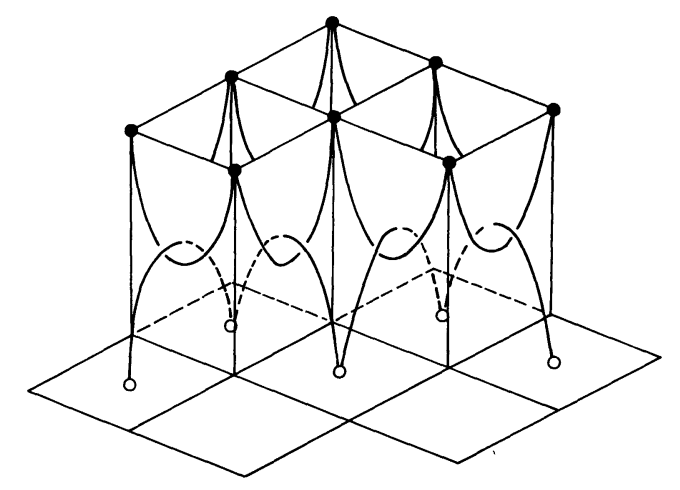

Fig. 12. - Three-dimensional structure of the locus of the cusps of the parabolic focal conics. (After Rosenblatt et al., Ref. [18].)

forms an array of defects which they interpreted as an array of parabolic focal conics (PFC's). In their model they described these defects in terms of layer curvatures, the geometry of the defects, the energetics of the defects and their mechanism of formation. Due to the well-defined layer thicknesses in smectic liquid crystals, any defects in the alignment of the layers are constrained to particular geometric configurations in which the smectic layers form a family of surfaces known as Dupin cyclides. The layers curve smoothly except at a series of line defects where cusps in the curvature of individual layers occur. Rosenblatt et al. suggested that the loci of the cusps are pairs of parabolas passing through each other's focus. An array of these PFC's forms the network visualized in figure 12. A consideration of the PFC model suggests the optical effects expected for a PFC array in a homeotropically aligned sample. The first feature to note in figure 12 is the presence of a three level system. The points where the parabola join together at the top or the bottom have four-fold symmetry. The layers curve away from the point where the parabolas join. In the middle, where the parabolas cross, the structure has the symmetry of a saddle surface. Rosenblatt $e t$ al. described the details of a model for this region in which the layers formed multiply connected surfaces. The details of this structure follow directly from the assumption that the elastic resistance to layer curvature is zero, while the resistance to change of layer thickness is infinite.

Figure $13 a$ is a qualitative representation of the layer deformation in a PFC array at four different heights in the sample. The cusps labeled $\mathrm{A}$ at the height labeled 1, where the four parabolas join, split into four different cusps at the height of 2 . The projections of the layer normals in the regions surrounding the cusps at height 1 radiate out from the cusps. This is illustrated in figure $13 b$. Similarly, at the lowest level, although the cusps are pointed up rather than down, the projections of the layer normals are radial with respect to the cusps. Intermediate heights 2 and 3 are more complicated since none of the four cusps at either height is cylindrically symmetrical. Furthermore, at the mid-height where the two parabolas 


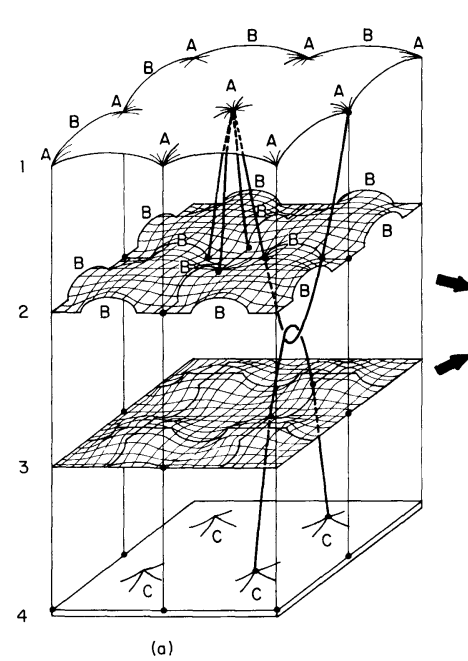

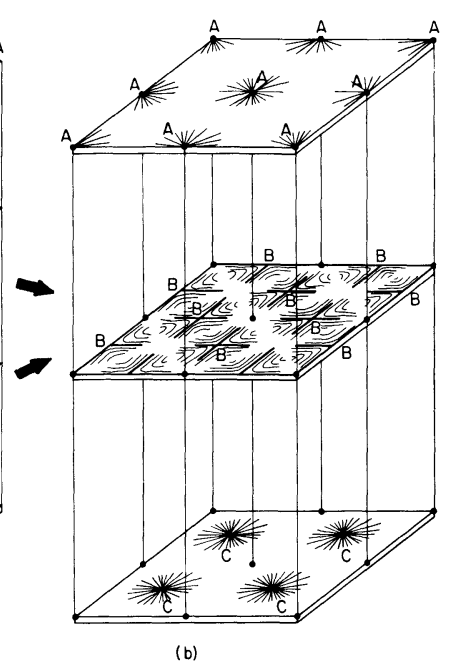

(b)

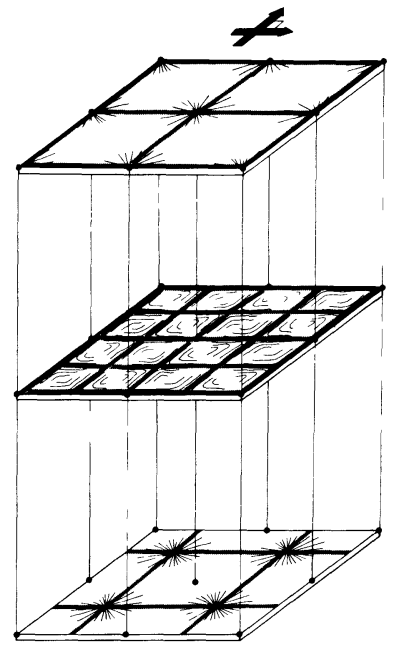

(c)

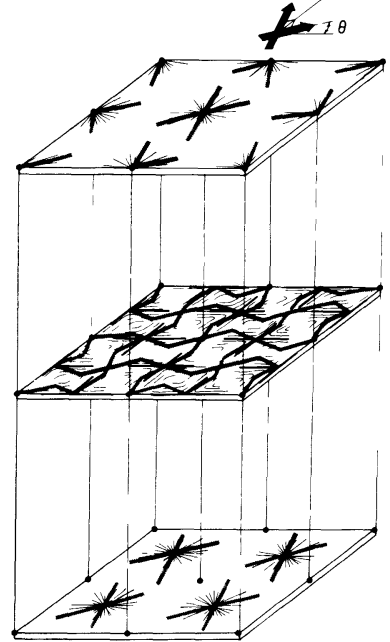

(d)

Fig. 13. - Representation of the smectic layer tilts in a PFC array and the effect of crossed polarizers. $a$ ) Three-dimensional view of the layer tilts at four different heights in the sample. Points labeled A are where the four parabolas join at the top. Points labeled B are where the parabolas cross. Points labeled $\mathrm{C}$ are where the four parabolas join at the bottom. $b$ ) Directions of biaxially induced by the tilts of the layers. c) Locus of light extinguished by the crossed polarizers oriented along the parabola. $d$ ) Locus of light extinguished by crossed polarizers rotated by an angle $\theta$ from $(c)$.

cross we cannot really represent the layers in a simple way. Nevertheless, the symmetry of the deformation at the mid plane can be inferred by averaging the layer tilts from levels 2 and 3 . The most important feature to note is that near the points $B$ the tilt directions are not radial. Figure $13 b$ illustrates the result for the average layer tilt directions. The local tilt direction is depicted by lines parallel to the projection onto a horizontal plane of the family of unit vectors normal to the layers. The tilt directions and their projections at the mid-height are derived from an averaging of the layer tilts from those layers between levels 2 and 3 of figure $13 a$. Figures $13 c$ and $13 d$ indicate the locus of points where light is extinguished when a PFC array is observed between crossed polarizers with a microscope that has a very short depth of focus. Focusing at three different heights obtains the three different patterns. When the crossed polarizers are parallel (perpendicular) to the projections of the normal to the layers, the polarizers lie along the local optic axes of the system ; incident light linearly polarized along the local optic axis is extinguished by the crossed polarizers. Thus, if the polarizers are oriented along the parabolas as in figure $13 c$ an array of black lines is expected to be observed oriented along the polarizer axis and running through the points where the parabolas intersect. The period of the top and bottom array is twice that of the middle array, and the intersections of the top array occur at the centre of the squares formed by the bottom array. If the crossed polarizers are rotated by some angle $\theta$ (Fig. 13d), the crosses at the top and bottom levels at points $\mathrm{A}$ and $\mathrm{C}$ will rotate parallel to the polarizers. However, those crosses centred at points $B$ in the middle array will rotate opposite to the polarizers.

Thus, the features predicted and observed for a
PFC array are : 1) If the focus is close to the height where the parabolas join at the top, an array of black lines occurs that intersects where the parabolas join. 2) When the focus is close to the height where parabolas join at the bottom, a similar array of lines with the identical periodicity occurs, except the intersections of these lines occur in the centres of the boxes formed by the top array. 3) If the focus is in the middle of the sample, the observed array has twice the periodicity of either the top or bottom array. 4) As the focus is shifted from the top to the middle of the sample, the locus of sharpest focus shifts from the point where the parabolas join at the top toward where the parabolas cross in the middle. The sharpest focus then shifts to the lower parabola, and as the focus is lowered further, the sharpness converges to where the parabolas join at the bottom. These observations are illustrated in figure 4.

Rosenblatt et al. suggested that the core region of a parabolic pair (i.e., the middle of the array) is energetically constrained to lie in the middle of the sample [18]. This is also consistent with the experimental observations. 5) If, while viewing either the top or bottom array, the polarizers are rotated by some angle $\theta$, the crosses centred where the parabolas join rotate with the crossed polarizers. 6) However, the crosses centred where the parabolas cross in the middle array are rotated by $-\theta$. Thus, the sharply focused cross in the middle array appears to rotate in the opposite direction. These results are illustrated in figure 5 .

The cusps in the layer curvature result in optical inhomogeneities along the parabolic lines that are expected to scatter light. On the other hand, since $n_{\mathrm{e}}-n_{0}$ is small for these lipids $[12,13]$, the scattered light will be weak where the cusps themselves are weak. In the PFC model proposed by Rosenblatt et al. 
large inhomogeneities only occur near the foci of the parabolas, i.e., where they cross. Thus, when viewed by dark field illumination, one expects to see only crosses at these spots. This prediction is borne out for the less birefringent arrays (Fig. 6).

The more birefringent arrays have a more complicated structure when viewed by dark field microscopy and cannot be explained in terms of the simple PFC model. Yet, these arrays have the same symmetry as the PFC arrays, and the pattern of crosses observed in dark field are still centred where the parabolas intersect in the PFC model. The additional structure may be due to additional defect structures existing within a pattern of PFC's and may serve as an additional mechanism to relieve stress and moderate layer curvatures. The more birefringent arrays always evolve from the less birefringent ones. Additionally, the more birefringent arrays have a period which is often a multiple of the period of the least birefringent array observed. The defects which appear in figures $8 a-8 e$ as concentric circles of dots may be due to the PFC cores and cusps from the original, smaller PFC's, or they may be due to additional defect structures.

The PFC model assumes a relatively simple defect structure in which the parabolas are the loci of individual cusps. We see no obvious reason why the parabolic lines could not be the loci of more complicated defect patterns. For example, there have been extensive theoretical descriptions of the different ways one might combine a number of focal conic defects to fill the space between two smectic regions which are misaligned with respect to each other [26-28]. The regular pattern of bright spots observed in figure 8 could possibly reflect a smaller substructure of focal conic defects that has been superimposed on the basic PFC arrays. The light and dark lines that seem to radiate out of the centre of the crosses in these arrays when viewed under crossed polarizers also suggest this.

The PFC model predicts that the maximum layer tilts should occur along the parabolas. Because the undisturbed smectic lipid liquid crystal has a positive uniaxiality $[12,13]$, the slow axis of the biaxiality due to tilts of the layers should be along the tilt. Measurements of the optical path difference support these predictions. However, these measurements also indicate that, except at the points where the parabolas join each other in the top and bottom arrays and where they cross each other in the middle array, the optical path difference along the parabola lines is almost constant. This is represented in figure $11 c$. The open (closed) dots are where the parabolas join at the bottom (top). The crosses are the points where the parabolas cross in the middle of the sample. A consideration of the detailed structure of the smectic layers of a PFC indicates that this is the expected result. Figure 14 shows the layer curvatures in the plane containing the parabola of a PFC array. The intersections between the smectic layers and the

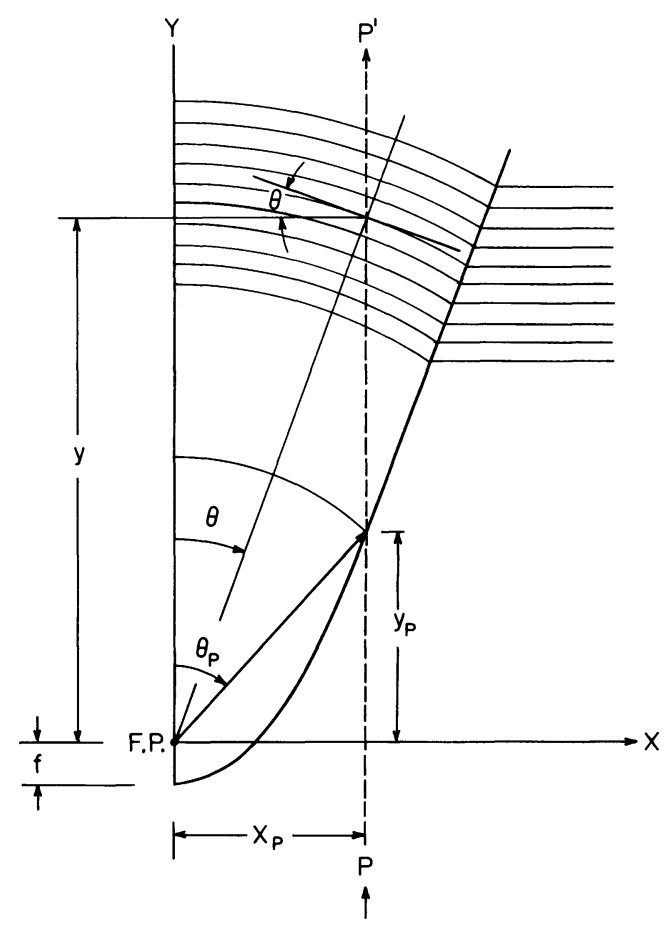

Fig. 14. - Parabola and layer curvatures in a PFC array.

planes containing the parabola form circles centred at the focal point FP, inside the parabola. Within this plane, and outside of the parabola, the layers are flat.

The optical path difference, $\Delta d$ for any ray linearly polarized at $45^{\circ}$ to this plane is :

$\Delta d \simeq \Delta n \int \sin ^{2} \theta \mathrm{d} y$,

where $\Delta n$ is the difference in the refractive indicies parallel and perpendicular to the smectic layers and $\theta$ is the angle the layers make witn the vertical ray. For the ray $\mathbf{P P}^{\prime}$ at a distance $X_{\mathrm{p}}$ from where the parabolas cross, the integral needs only to be evaluated from $y_{\mathrm{p}}$ to $\infty$ since the layers are flat below $y_{\mathrm{p}}$.

$\Delta d \simeq \Delta n X_{\mathrm{p}} \theta_{\mathrm{p}}=2 \Delta n f\left[s \tan ^{-1} \frac{2 s}{s^{2}-1}\right]$,

where $f$ is the focal length of the parabola and $s=X_{\mathrm{p}} / 2 f$. For values of $s \gtrsim 3$, which excludes the regions where the parabolas cross, $\Delta d$ is almost constant and varies less than $4 \%$. For the polygonal array in figure $4, f$ can be calculated to be about $1 \mu \mathrm{m}$. For $s=3$ or $X_{\mathrm{p}}=6 \mu \mathrm{m}, \theta_{\mathrm{p}}$ is calculated to be 0.64 radians. For $\Delta n$ equal to about 0.02 [12, 13], the calculated $\Delta d \simeq 76 \mathrm{~nm}$ is in good agreement with the measured value of $80 \mathrm{~nm}$. The undulations in $\Delta d$ observed for the very birefringent arrays such as in figure 7 reflect additional layer curvatures not described in the PFC model.

The production of polygonal arrays by temperature jumps has been observed previously in thermotropic smectic liquid crystals $[17,29]$. For DPPC with $7 \%$ 
water at $80^{\circ} \mathrm{C}$ the PFC arrays form upon temperature increases of less than $1^{\circ} \mathrm{C}$. For a sample $125 \mu \mathrm{m}$ thick with a linear coefficient of thermal expansion normal to the layers of $-2 \times 10^{-3}{ }^{\circ} C^{-1}[30,31]$, this would correspond to an equivalent dilation of less than $0.25 \mu \mathrm{m}$. For an $80 \mu \mathrm{m}$ thick DLPC sample at room temperature containing $20 \%$ water by weight, temperature increases of $4{ }^{\circ} \mathrm{C}$ are required to form the PFC arrays. Assuming a similar temperature coefficient of expansion, this represents an equivalent dilation of about $0.60 \mu \mathrm{m}$. However, preliminary experiments using a piezoelectric device to dilate DLPC samples with $20 \%$ water indicate that at room temperature a mechanical dilation of $2.0 \mu \mathrm{m}$ will barely form an observable PFC structure. Unless DLPC has a coefficient of thermal expansion that is approximately three times smaller than that for DPPC, we do not understand the difference between the mechanical and temperature induced dilations.

Although Rosenblatt et al. were not able to formulate a well defined theoretical representation of the formation of PFC arrays, they observed that for the material they studied, CBOOA, the arrays formed spontaneously at dilations $\delta t_{\mathrm{PFC}} \gtrsim 3 \pi \lambda$ where $\lambda=\sqrt{K \mid B} ; K$ is the Frank elastic constant for splay and $B$ is the smectic layer compressional elastic constant. In the case of CBOOA and other thermotropic liquid crystals, if one is not near a critical temperature, $\lambda$ is roughly equal to the thickness of a smectic layer [32, 33]. Assuming this is also true for the lipid-water liquid crystals we have studied, their argument implies PFC arrays should form at dilations of the order of $500 \AA$. We have never observed PFC arrays for thermally induced equivalent dilations less than $0.15 \mu \mathrm{m}$ and for mechanical dilations of less than $\sim 2 \mu \mathrm{m}$.

Rosenblatt et al. argued that for dilations of the order of $\delta t_{\mathrm{PFC}}$, the arrays should have focal lengths of the order of either $\delta t_{\mathrm{PFC}} / 2$ or $\delta t_{\mathrm{PFC}} / 6$. If we accept the estimate of $\delta t_{\mathrm{PFC}} \sim 3 \pi \lambda$ and take $\lambda \sim$ a single smectic layer thickness, the focal length should be either $\sim 25 \mathrm{~nm}$ or $8 \mathrm{~nm}$. This is considerably less than the minimum focal lengths we have observed. For example, if the parabolas in a PFC meet at a height $d$ above the mid-height of the sample, the half width of any one parabola at this height is approximately given by $R^{2}=4 d f$, where $f$ is the focal length of the parabolas. Typically, we observe values of $R$ of the order of $10 \mu \mathrm{m}$ when $d \simeq 20 \mu \mathrm{m}$, implying $f \simeq 1 \mu \mathrm{m}$.

If their suggested values for $\delta t_{\mathrm{PFC}}$ were correct, it would have been very difficult for us to actually see these small arrays. We previously argued that the optical path differences for different polarizations were no greater than something of the order of $4 \Delta n f$. If $\delta t_{\mathrm{PFC}} \sim 25 \mathrm{~nm}$ and $f \simeq 12.5 \mathrm{~nm}$ and $d \lesssim$ half the sample height, which is approximately $100 \mu \mathrm{m}, R$ cannot be greater than $\sim 2 \mu \mathrm{m}$. Taking $\Delta n \simeq 0.02$, the expected path length difference is of the order of $1 \mathrm{~nm}$.
This PFC would not have been detected in our samples, while in CBOOA, where $\Delta n \sim 0.4$ [34], the same defect would have a detectable path difference of $20 \mathrm{~nm}$.

On the other hand, if we assume that $\delta t_{\mathrm{PFC}}$ corresponds to the minimum temperature induced equivalent dilation that will produce a detectable $\mathrm{PFC}$ in DPPC, $\delta t_{\mathrm{PFC}} \simeq 0.15 \mu \mathrm{m}$. Taking $\delta t_{\mathrm{PFC}} \sim 3 \pi \lambda$ results in $\lambda \simeq 15 \mathrm{~nm}$, which is not unreasonable. Unfortunately, if we accept the argument that $f \sim \delta t_{\mathrm{PFC}} / 2$ or $\delta t_{\mathrm{PFC}} / 6$, we predict focal lengths that are much smaller than the observed values.

The only possibility that might remove what otherwise appear to be irreconcilable conflicts between our results and the relations proposed by Rosenblatt et al. is that, as mentioned above, we only observe PFC arrays for dilations $\delta t \gg \delta t_{\mathrm{PFC}}$. For DPPC we observe $f \sim 1 \mu \mathrm{m}$ when the thermally induced, equivalent $\delta t \gtrsim 0.15 \mu \mathrm{m}$. For DLPC we do not actually know the coefficient of thermal expansion; however, a mechanical $\delta t \sim 2 \mu \mathrm{m}$ produces an observable array with $f \sim 1 \mu \mathrm{m}$. Rosenblatt et al. suggest a half width $R \sim \sqrt{(\delta t) t}$ when $\delta t \gg \delta t_{\mathrm{PFC}}$. Our results are in reasonable agreement with this and we do observe small increases in $R$ with increases of $\delta t$. On the other hand, at a certain point we observe a doubling of $R$ and additional structure beyond that contained in the PFC model. Thus, it is not clear what one should expect for $R$ when $\delta t \gg \delta t_{\mathrm{PFC}}$.

The PFC arrays were suggested by Rosenblatt et al. [18] to evolve from the undulation instability. Aside from the criterion for PFC formation, the response of smectic liquid crystals to dilative strains has been understood for some time. Below some critical strain the layers merely dilate. However, for strains larger than some threshold, $t_{\mathrm{c}}$, the layers begin to undulate $[32,35]$. This undulation phenomenon has been observed by light scattering from lipid liquid crystals of DPPC [13]. However, experiments using piezoelectric elements to compress and dilate the glass plates enclosing samples of DLPC with $20 \%$ water, suggest that the PFC's form not only by dilation, but also by compression of the sample. This is difficult to reconcile with the explanation based on the undulative instability. Since we have never observed the PFC array to form by a decrease in the temperature of the sample, further work is necessary to understand the formation of PFC's by compression.

Polygonal arrays, qualitatively similar to those observed for the lipids, have been observed by Bouligand [15] in cholesteric liquid crystals. The geometry suggested by Bouligand for the polygonal arrays differs from the PFC structure by allowing large layer tilts and not requiring those layers next to the glass surfaces to be parallel to them. This may be allowed for cholesterics due to the weak anchoring of the cholesteric helix at the glass surfaces. However, in 
the lipids and in CBOOA [18] the anchoring appears to be strong.

The PFC arrays in the lipids appear more regular than those in CBOOA. Also, the appearance and disappearance of the arrays shows a more cooperative behaviour than in CBOOA; the lipid PFC's rarely appear and disappear individually. Also, they are stable for long periods of time. The differences between the PFC's in CBOOA and in the lipids may be due to differences in the smectic layer structures of thermotropic and lyotropic liquid crystals. Due to the separate polar and non-polar parts of the lipid molecules and because of the water layers between the lipid bilayers, the smectic layers of the lipid are very well defined. There is little permeation of a molecule from one lipid bilayer to another; as a result, it is difficult for the smectic layers in lipids under stress to break and reform into configurations of lower energy.

5. Conclusions. - We have suggested that some of the polygonal arrays found in lipid liquid crystals of phosphatidylcholines are parabolic focal conic arrays. Other more birefringent arrays have a more complex structure. Since all of the polygonal arrays appear similar when viewed between crossed polarizers, they can only be differentiated by additional techniques such as dark field microscopy. These arrays are ubiquitous in lipid liquid crystals and result from temperature increases and mechanical strains. Careful thermostating of these liquid crystals is necessary if one hopes to maintain aligned, uniaxial and defect-free samples.

Acknowledgments. - We would like to gratefully acknowledge very helpful conversations with Robert Meyer and Y. Bouligand. We would also like to acknowledge the helpful comments and criticisms by Charles Rosenblatt and Michael Fisch. We thank Paul Demchak for technical assistance in the early stages of this work. In addition, one of us (SA) would like to thank Robert Meyer for his encouragement in the early stages of this work.

\section{References}

[1] Chapman, D., Quart. Rev. Biophys. 8 (1975) 185.

[2] Melchior, D. L. and Stein, J. M., Ann. Rev. Biophys. Bioeng. 5 (1976) 205.

[3] Lepesant, J. P., Powers, L. and Pershan, P. S., Proc. Natl. Acad. Sci. (USA) 75 (1978) 1792.

[4] Chan, W. and Pershan, P. S., Phys. Rev. Lett. 39 (1977) 1368.

[5] Wu, E.-S., Jacobson, K. and Papahadjopoulos, D., Biochem. 16 (1977) 3936.

[6] Sanson, A., Ptak, M., Rigaud, J. L. and Gary-Bobo, C. M., Chem. Phys. Lipids 17 (1976) 445.

[7] Jost, P., Libertini, L. J., Hebert, V. C. and Griffith, O. H., J. Mol. Biol. 59 (1971) 77.

[8] LeVine, Y. K. and Wilkins, M. H. F., Nature New Biol. 230 (1971) 69.

[9] Hsia, J.-C., Schneider, H. and Smith, I. C. P., Biochem. Biophys. Acta 202 (1970) 399.

[10] Libertini, L. J., Waggoner, A. S., Jost, P. C. and GrifFiTh, O. H., Proc. Natl. Acad. Sci. (USA) 64 (1969) 13.

[11] Levine, Y. K., Bailey, A. J. and Wilkins, M. H., Nature 220 (1968) 577.

[12] Powers, L. and Pershan, P. S., Biophys. J. 20 (1977) 137.

[13] Powers, L. and Clark, N. A., Proc. Natl. Acad. Sci. (USA) 72 (1975) 840.

[14] Asher, S. A. and Pershan, P. S., Biophys. J. 21 (1978) 123-a.

[15] Bouligand, Y., J. Physique 33 (1972) 715.

[16] Kahn, F. J., Appl. Phys. Lett. 3 (1973) 111.

[17] Ribotta, R., Ph. D. thesis, Université de Paris-Sud, Orsay; J. Physique Colloq. 37 (1976) C3-149.

[18] Rosenblatt, C. S., Pindak, R., Clark, N. A. and Meyer, R. B., J. Physique 38 (1977) 1105

[19] Elworthy, P. H., J. Chem. Soc. (1959) 813 and (1960) 139.
[20] Luzzati, V. and Tardieu, A., Ann. Rev. Phys. Chem. 25 (1974) 79.

[21] Chapman, D., Williams, R. M. and Ladbrooke, B. D., Chem. Phys. Lipids 1 (1967) 445.

[22] Williams, R. M. and Chapman, D., Progress in the Chemistry of Fats and Other Lipids, R. T. Holman, editor (Pergamon Press, Oxford) 1970, Vol. 11, p. 1.

[23] Luzzati, V., Biological Membranes, D. Chapman, editor (Academic Press, London) 1968, Vol. 1, p. 71.

[24] Ranck, J. L., Mateu, L., Sadler, D. M., Tardieu, A., Gulik-KrzYwicki, T. and Luzzati, V., J. Mol. Biol. 85 (1974) 249.

[25] Luzzati, V., Mustacchi, H., Stoulios, A. and Jusson, F., Acta Crystallogr. 13 (1960) 660.

[26] Bideaux, R., Boccara, N., Sarma, G., De Seze, L., De Gennes, P. G. and Parodi, O., J. Physique 34 (1973) 661.

[27] Bouligand, Y., J. Physique 33 (1972) 525.

[28] BraGG, W. H., Nature 133 (1934) 445.

[29] Ribotta, R., J. Physique Colloq. 37 (1976) C3-149.

[30] Luzatti, V., Biological Membranes (Academic Press, New York) 1968, p. 71.

[31] Rand, R. P. and Pangborn, W. A., Biochem. Biophys. Acta 318 (1973) 299.

[32] Delaye, M., Ribotta, R. and Durand, G., Phys. Lett. 44A (1973) 139.

[33] DE Gennes, P. B., The Physics of Liquid Crystals (Oxford University Press, London) 1974, p. 291.

[34] Huang, C. C., Pindak, R. S. and Ho, J. T., J. Physique Lett. 35 (1974) L-185.

[35] Clark, N. A. and Meyer, R. B., Appl. Phys. Lett. 22 (1973) 4. 